\title{
Millimeter Wave Wireless System Based on Point to Multipoint Transmissions
}

\author{
Claudio Paoloni ${ }^{1}$, François Magne ${ }^{2}$, Frédéric André ${ }^{3}$, Viktor Krozer ${ }^{4}$, Rosa Letizia ${ }^{1}$, Marc Marilier ${ }^{5}$, \\ Antonio Ramirez ${ }^{6}$, Marc Rocchi ${ }^{5}$, Ruth Vilar ${ }^{7}$, Ralph Zimmerman ${ }^{8}$ \\ ${ }^{1}$ Lancaster University, Lancaster LA1 4YW, UK \\ email: c.paoloni@lancaster.ac.uk \\ ${ }^{2}$ When-AB, Paris, France \\ ${ }^{3}$ Thales Electron Devices, Velizy 78140, France \\ ${ }^{4}$ Goethe University of Frankfurt, Frankfurt 60323, Germany, \\ ${ }^{5}$ OMMIC S.A.S., Limeil Brevannes 94453, France \\ ${ }^{6}$ Fibernova Systems, Valencia 46022, Spain \\ ${ }^{7}$ Universitat Politècnica de València, Valencia 46022, Spain, \\ ${ }^{8}$ HF Systems Engineering GmbH, Kassel 34123, Germany
}

\begin{abstract}
The continuously growing traffic demand has motivated the exploration of underutilized millimeter wave frequency spectrum for future mobile broadband communication networks. Research activities focus mainly on the use of the $\mathrm{V}$ band $(59-64 \mathrm{GHz})$ and E-band $(71-76 \& 81-84 \mathrm{GHz})$ to offer multi-gigabit point to point transmissions. This paper describes an innovative $\mathrm{W}$-band $(92-95 \mathrm{GHz})$ point to multipoint wireless network for high capacity access and backhaul applications. Point to multipoint wireless networks suffer from limited RF power available. The proposed network is based on a high power, wide band traveling wave tube of new generation and an affordable high performance transceiver. These new devices enable a new transmission paradigm and overcome the relevant technological challenges imposed by the high atmosphere attenuation and the presently lack of power amplification required to provide adequate coverage at millimeter waves.
\end{abstract}

Keywords-millimeter wave; backhaul; access; 5G; traveling wave tube; MMIC;

\section{INTRODUCTION}

To satisfy the demand of high capacity everywhere is the formidable challenge of the new generation networks. This is not only a priority for implementing the future $5 \mathrm{G}$ infrastructures, but also for the whole distribution of highspeed Internet in different scenarios such as residential and business access.

Fiber is widely viewed as a time-proven technology that offers almost limitless capacity and scalability. Unfortunately, the fiber cannot be deployed everywhere, for its high deployment cost, and excessive logistical challenges. As a result, large percentage of territories and consequently of users are in a sort of digital divide conditions. Due to these

The project has received funding from the European Union's Horizon 2020 research and innovation program under grant agreement no 644678 . drawbacks, wherever the fiber is not deployable, wireless networks are well perceived as a mandatory complement of the fiber, as they reduce cost and improve the speed of deployment.

Looking ahead to next generation of wireless networks, there are different approaches proposed to increase the network capacity. One of the most promising solutions to satisfy the need of multi-gigabit transmission is the use of the spectrum in millimeter wave range. Indeed, the wide relative bandwidth associated to this frequency range can potentially support capacity not far from that of a typical fiber. Consequently, millimeter wave (mmWave) communications are a key enabler for the forthcoming $5 \mathrm{G}$ networks, small cell backhaul and broadband access [1].

In recent years, a relevant research activity mainly focused on the use of the V-band $(59-64 \mathrm{GHz})$ and E-band $(71-76 \&$ $81-84 \mathrm{GHz}$ ) is in progress worldwide [2]. Both millimeter wave bands offer great capacity, but are only used in point to point (PtP) configurations (e.g., E-band is regulated only for PtP links). This results in innately equipment-heavy networks and expensive total cost of ownership (TCO). For this reason, point to multipoint (PmP) systems are preferred over PtP from a TCO point of view. Moreover, PmP architecture can efficiently allocate capacity among different endpoints, resulting in a flexible network topology that lends itself to subscriber adds, moves and changes, thus lowering operational expenditures (OPEX) and facilitating rapid subscriber acquisition.

The W-band $(92-95 \mathrm{GHz})$ is a lightly regulated portion of the spectrum, open for PmP communications, that will open new opportunities for multi-gigabit transmissions, enabling 
cost-effective access and backhaul. In this paper, the approach to realize the first PmP system at W-band as proposed in the EU Horizon 2020 TWEETHER project will be described [3]. In particular, the TWEETHER project is developing a new concept of front-end radio based on a novel W-band vacuum electron device and a high performance transceiver. The availability of such devices at $\mathrm{W}$-band will foster a new network paradigm whose objective is to overcome the digital divide due to the lack of high speed connection in absence of the fiber and to pave the way towards the development of new high density cell structure of future $5 \mathrm{G}$ networks.

\section{W-BAND TWEETHER SYSTEM}

\section{A. Network architecture}

For cost-effective mobile backhaul and fixed access deployments, the TWEETHER vision is to develop a hybrid network architecture based on three tiers (Fig. 1):

- Tier 1 based on fiber for large aggregated capacity.

- Tier 2 conceived as a wireless mmWave subnetwork for high capacity distribution.

- $\quad$ Tier 3 representing the final distribution based on LTE technology and on $5 \mathrm{G}$ in the future.

The use of the best technology in each tier allows maximizing the capacity and minimizing the deployment cost.

In particular, the TWEETHER system addresses the middle tier of this hybrid network concept, which distributes the high capacity coming from the fiber to a number of small cells or residential access Point of Presences (PoP), each receiving up to $200 \mathrm{Mbps}$. This capacity can either be dedicated to a small cell or shared in the case of access solutions for consumers and enterprises. This flexibility in capacity provisioning is obtained using a new multiplexing technology that allows the aggregation of multiple independent channels (modems) through wideband radios and antennas onto a single air interface.

The TWEETHER system is a PmP W-band network composed of a certain number of transmission hubs $(\mathrm{TH})$ to cover a region. The service area covered by a $\mathrm{TH}$ is divided in sectors which allow efficient coverage, and frequency reuse. Each sector supports a sub-net that distributes the capacity to the number of terminals to be fed, mainly in Line-of-Sight (LOS) conditions (Fig. 2). The first implication of giving service over a wide area is that, differently from narrow beam antennas used in PtP links, a low directive antenna is required. Therefore, to provide the same energy level at the receiver, the output power at the transmission hub in a PmP configuration must be substantially higher.

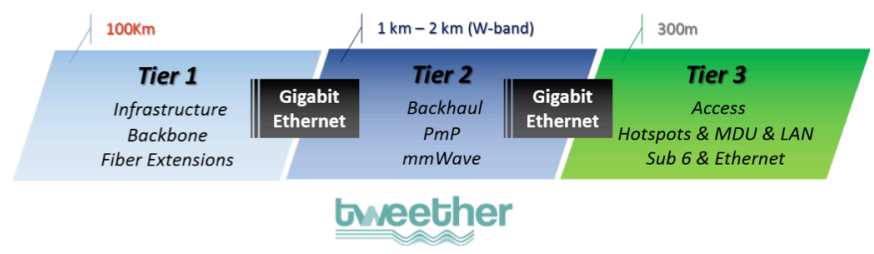

Fig. 1 The TWEETHER system to bridge Tier 3 to Tier 1.
However, the atmospheric attenuation in rain condition is one of the main obstacles for propagation at millimeter wave frequency and is a key factor to be considered for the design of a W-band system [4]. Therefore, the requirement of low directive antennas for PmP configurations together with the high attenuation in the millimeter wave region have to be taken into account to derive a realistic value of output power at the transmitter to enable $\mathrm{W}$-band PmP communications.

\section{B. Capacity and coverage scenario at $W$-band}

The target of TWEETHER is to provide a capacity of 10 $\mathrm{Gbps} / \mathrm{km}^{2}$ in a circular region with a radius in the order of 1 $\mathrm{km}$, assuring an availability of the $99.99 \%$ for two main applications: small cell backhaul and broadband access.

Two scenarios can be considered on the basis of capacity and coverage requirements:

\section{Scenario 1: Small cell Backhaul \\ - 1000 mobile users, \\ - 30 to 50 small cells with 100 m radius, \\ - 5 -10 Mbps per mobile user.}

\section{Scenario 2: Broadband Access}

- 100 houses or 25 enterprises,

- $50-200 \mathrm{Mbps}$ per fixed access PoP.

To satisfy the requirements of these two scenarios, the radio and antenna parameters have to be computed to ensure the required coverage, and the expected capacity and throughput on each segment of the network. A coverage model has been implemented and used to determine the specifications of the transmission hub and the terminals for $10 \mathrm{Gbps} / \mathrm{km}^{2}$ in $1-\mathrm{km}$ radius area:

- Low antenna gain at the transmission hub (TH): about $18 \mathrm{dBi}$.

- $\quad$ Directive antenna at the terminal: $35 \mathrm{dBi}$.

- $\quad$ Required TH bandwidth: 500 MHz.

- $\quad$ Required bandwidth at the terminal: $\sim 40 \mathrm{MHz}$.

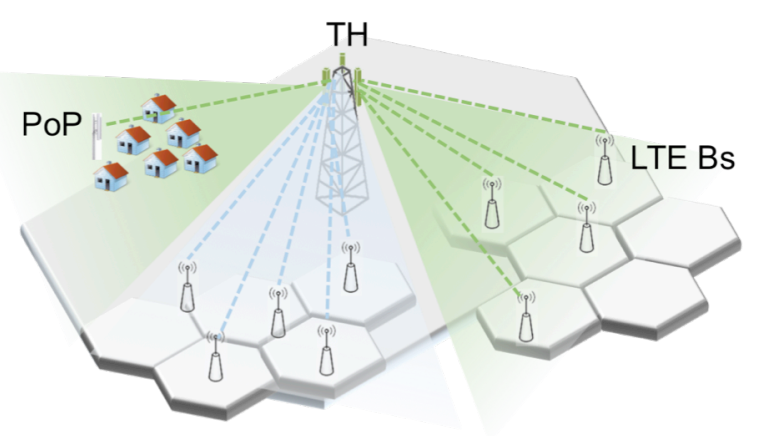

Fig.2 PmP network composed of a transmission hub (TH) with several sectors, giving service to several LTE base stations and access PoP located in a wide area. 
The output power specifications are:

- $1 \mathrm{~W}$ (saturated) for the terminal,

- $40 \mathrm{~W}$ or $46 \mathrm{dBm}$ (saturated) for the TH.

It is notable that the main technological challenge in $\mathrm{W}$ band to enable the proposed wireless systems is to satisfy the need of power at the terminal and at the transmission hub, as commented previously.

State of the art high power solid-state MMIC processes could provide power amplifiers with saturated power in the range of $30 \mathrm{dBm}[5][6]$. On the contrary, no amplifier with a saturated power level in the range of $40 \mathrm{~W}$ and sufficient bandwidth is available today for wireless communication systems at millimeter waves. The TWEETHER project is addressing this challenge by introducing a novel technological solution, beyond the state-of-the-art, which is based on vacuum electron devices.

\section{ENABLING TECHNOLOGY FOR W-BAND TWEETHER SYSTEM}

\section{A. W-band Traveling Wave Tube}

The computation of the power requirement to enable a PmP transmission reveals the great challenge of millimeter wave wireless communications. Microwave and millimeter wave PtP wireless systems presently rely on solid-state amplifiers [5]. While at microwaves, transmission power in the order of hundreds of Watt is easily achieved, at millimeter wave state of the art power amplifiers are limited to a few Watt of saturated power. Further, with the increase of the frequency from, e.g, Qband to $\mathrm{W}$-band, the output power decreases according to the well-known relationship that scales the output power by $20 \mathrm{~dB}$ per decade.

Vacuum electronics is the oldest electronic discipline that first enabled wireless communications and the first computers (ENIAC). One of the most common vacuum electron device is the Traveling Wave Tube (TWT), presently used in most communications satellites as wideband power amplifier for its high RF power levels and reliability [7][8].

The TWT (Fig. 3) main parts are the electron gun, the slow wave structure (SWS), the collector, the magnetic focusing system and the radiofrequency (RF) vacuum window. The electron gun generates an electron beam of given current and electron energy by using a thermionic cathode. The slow-wave structure (SWS) (or interaction structure) is a periodic waveguide that slows down the phase velocity of the RF field to a speed lower than the speed of light to permit in-phase interaction with the electron beam traveling along the structure. The magnetic focusing system keeps the electron beam confined throughout the slow-wave structure. The collector recovers the energy of the electrons in the beam at the end of the tube to increase the operational efficiency. The RF vacuum window maintains the vacuum inside the tube, but is transparent to the RF field to permit the power transfer with the external flanges.

In contrast to solid-state devices, where the current flows in the crystalline reticule suffer from collision effects that

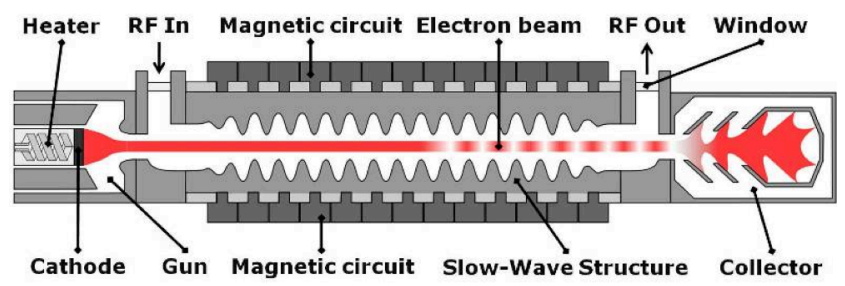

Fig. 3 Traveling wave amplifier schematic

translate into thermal dissipation, in the TWT the current flows in the vacuum without any perturbation. This permits to build TWTs with operation frequency limited, in principle, only by the availability of microfabrication techniques to realize SWS with dimensions suitable to support the operating frequency of interest. Vacuum electronic devices have indeed been proved up to $1000 \mathrm{GHz}(1 \mathrm{THz})$ [9].

A novel W-band TWT, is the enabling device for the Wband wireless network concept of the Horizon 2020 TWEETHER project (see Fig. 2). It will be integrated at the transmission hub to guarantee the proper output power for the required capacity and range. The TWT specifications, derived by an accurate link budget model, are $40 \mathrm{~W}$ saturated RF output power and more than $40-\mathrm{dB}$ gain in the $92-95 \mathrm{GHz}$ range, with high linearity. Easy fabrication, high power efficiency, long lifetime, compact dimensions in the order of a few $\mathrm{dm}^{3}$, low weight and low cost are important constraints considered in the design.

The challenge is demanding due to the convergence of two conflicting requirements: high technology approach for performance and low cost for massive productions for a largescale deployment of the system. The design strategy is focused on defining interaction structures of very simple fabrication to reduce the assembly issues, relaxing the gun specifications, and

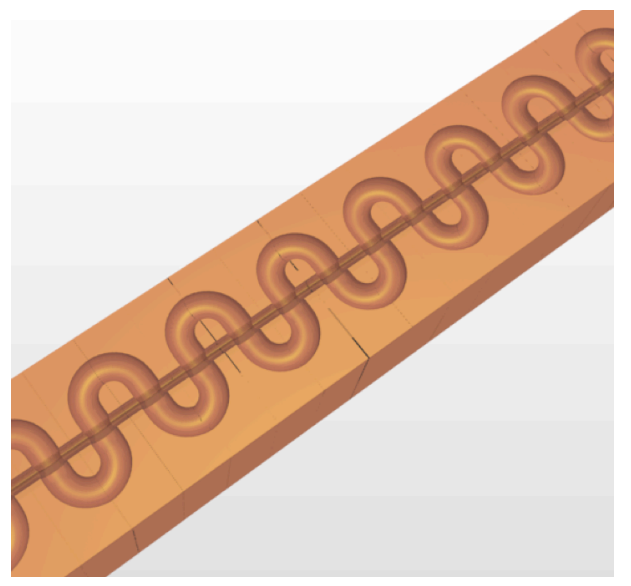

Fig. 4 Longitudinal Section (one half) of folded waveguide with the beam tunnel (red)

to use low cost components [10][11]. A first prototype satisfying the requirement is in advanced fabrication status. 
The helix is the most used slow wave structure for wideband TWTs for communications, in a wide range of microwave frequencies Error! Reference source not found.. The fabrication of helices in the millimeter wave range, above 60 $\mathrm{GHz}$, is impracticable due to the very small dimensions (a few hundred micron diameters). Therefore, different full metal structures capable of supporting the $\mathrm{W}$-band signal have to be adopted.

The folded waveguide (FWG) (Fig.4) is a rectangular waveguide folded with a defined period crossed by a beam tunnel where the electron beam flows. This interaction structure has been chosen due to its suitable compromise between performance and fabrication challenge [12]. The fabrication is performed by CNC-milling, in two halves perfectly symmetrical that has to be precisely assembled. The total length of the structure, based on the FWG to produce the $40-\mathrm{W}$ output power and $40-\mathrm{dB}$ gain, is about $11 \mathrm{~cm}$. It requires a focusing magnetic field less than $0.3 \mathrm{~T}$. The simulations were performed by 3-D electromagnetic simulators and particle in cell simulators, specific for vacuum electron devices simulations. As shown in Fig. 5, the FWG TWT gain obtained with this design is over $40 \mathrm{~dB}$.

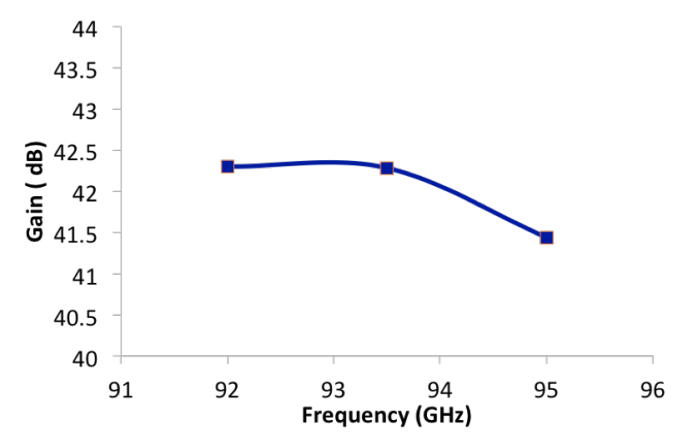

Fig. 5 FWG TWT Gain

\section{B. W-band electronics}

As described in the previous sections, the main novelty of TWEETHER system is in the transmission hub (Fig. 6), where a TWT is used to provide the required output power. However, due to the very high frequency range adopted the challenge is not limited to the TWT.

The W-band electronics is still at an early stage and relevant advancements are required in the solid-state circuit design and fabrication as well as in integration procedures. In particular, the availability of $\mathrm{W}$-band radio chipsets with high performances is crucial to fulfill the system requirements in

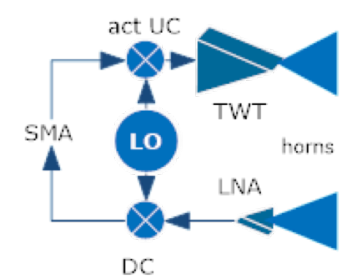

Fig. 6 Schematic of the Transmission Hub

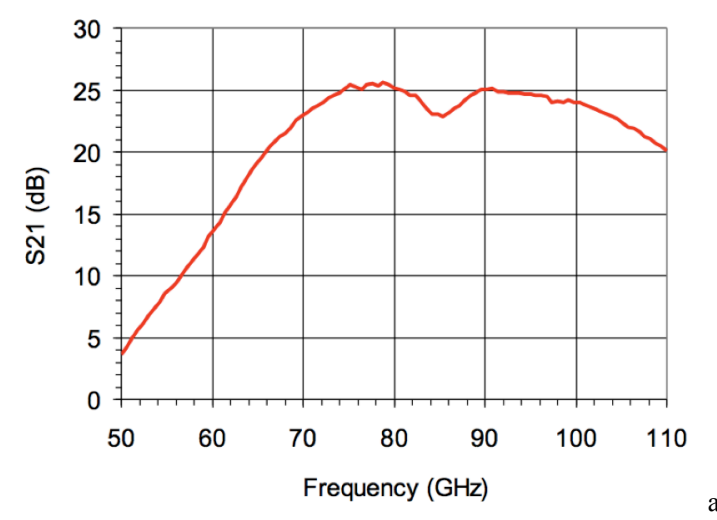

a)

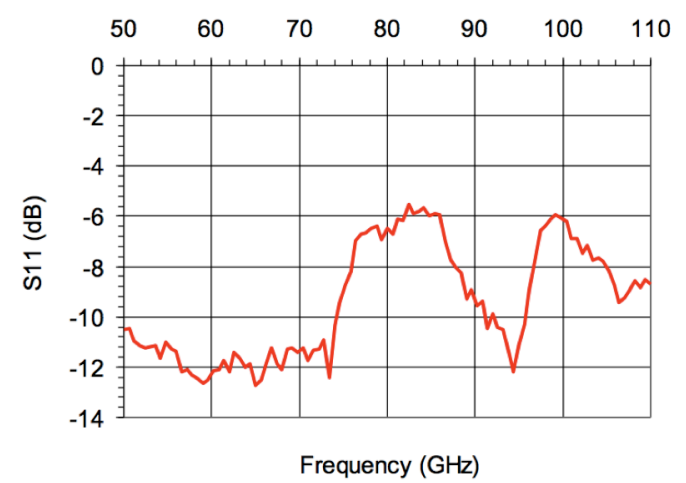

b)

Fig.7 W-band MMIC Low Noise amplifier (OMMIC). a) Gain; b) Return loss

terms of coverage and capacity defined for the target scenarios. Therefore, the chipset operating at W-band is the second enabling group of components for the TWEETHER system. This Monolithic Microwave Integrated Circuits (MMIC) chipset includes an $\mathrm{x} 8$ frequency multiplier, the upconverter, the down-converter, the low noise amplifier (gain and return loss are shown in Fig.7) and the power amplifier. The same chipset is used for the transmission hub and the terminal, resulting in a substantial reduction of production cost.

The most critical component of the chipset is the power amplifier, as it is fundamental for the terminal return path performance. A $\mathrm{P}_{\text {sat }}$ of about $23 \mathrm{dBm}$ is required to support the required range. The first samples of MMIC power amplifiers have been successfully fabricated and measured. The layout is shown in Fig.8a. The gain, output power and efficiency are shown in Fig. 8 b.

\section{W-band front-end modules}

The different technologies (vacuum electronics and chipset) will be seamlessly assembled in a single front-end. The Wband front-end modules for the terminals and the transmission hub will consist of three main stages: 
The packaged TWT and power supply for the hub. The air interface will be ensured by a sector horn
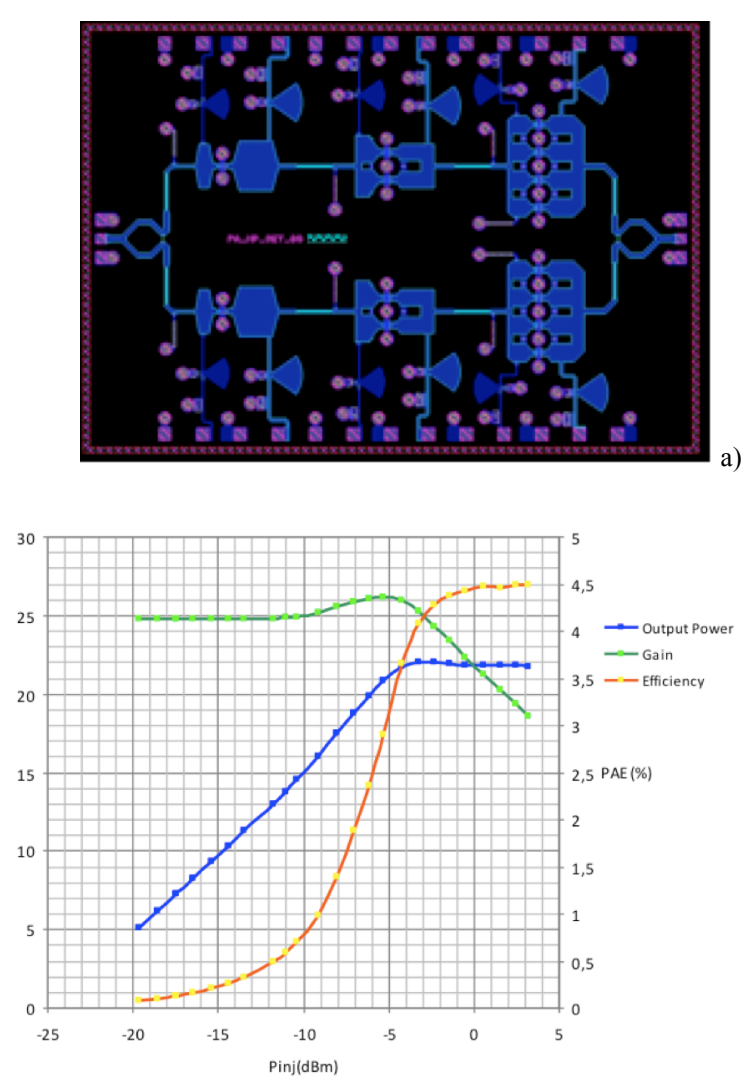

b)

Fig.8 W-band MMIC power amplifier (OMMIC). a) Layout; b) Output power, Gain and Power Added Efficiency

antenna, directive in elevation. The interface with the TWT will be realized by waveguide.

- $\quad$ Packaged Transmitter and Receiver hub module with synthesizer and synchronization function. RF interfaces will be connected with waveguides to the TWT and to the receiver horn antenna.

- $\quad$ Packaged Transceiver radio module for the terminal will comprise an RF board on which all the MMICs will be integrated. This RF board will be packaged and plugged on a motherboard which will include the IT functions such as synthesizer, IF buffers and power supplies.

\section{CONCLUSIONS}

The TWEETHER system will be the first W-band (92-95 $\mathrm{GHz}$ ) system for point to multipoint access and backhaul. It is designed to provide up to $10 \mathrm{Gbps} / \mathrm{km}^{2}$ on sectors with aperture of $90^{\circ}$ or less with at least $1-\mathrm{km}$ radius coverage.

A wide range of coverage scenarios could be implemented depending on the nature of the area to be covered. The high flexibility of TWEETHER system will open a new approach in the definition of both dense or low density networks. The millimeter wave electronic components to enable this new transmission paradigm are in advanced phase of design and fabrication.

\section{REFERENCES}

[1] T.S. Rappaport et al., " Millimeter Wave Mobile Communications for 5G Cellular: It will work!”, IEEE Access, pp. 335-349, Mai 2013.

[2] C. Colombo; M. Cirigliano "Next-generation access network: A wireless network using E-band Radio frequency (71-86 Ghz) to provide wideband connectivity," in Bell Labs Technical Journal, vol.16, no.1, pp.187-205, June 2011.

[3] TWEETHER website [Online]. Available: http://www.tweether.eu

[4] ITU-R P.838-3, "Specific attenuation model for rain for use in prediction methods," 2005.

[5] A. Brown, K. Brown, J. Chen, K. C. Hwang, N. Kolias, and R. Scott, "W-Band GaN Power Amplifier MMICs," in 2011 IEEE MTT-S International Microwave Symposium Digest (MTT), pp. 1-4, Jun. 2011.

[6] J. M. Schellenberg, "A 2-W W-Band GaN Traveling-Wave Amplifier With 25-GHz Bandwidth," in IEEE Transactions on Microwave Theory and Techniques, vol. 63, no. 9, pp. 2833-2840, Sept. 2015.

[7] C. K. Chong and W. L. Menninger, "Latest Advancements in HighPower Millimeter-Wave Helix TWTs," IEEE Trans. on Plasma Science, vol.38, no.6, pp.1227,1238, June 2010.

[8] D. Kupidura, F. Vasseur, A.Laurent, P. Ehret, E. Bosch, "Thales 45W and 100W Q-Band Conduction Cooled Travelling Wave Tubes" Proc. IEEE 16th Int. Vac. Electron. Conf. (IVEC), 2015.

[9] C. Paoloni, A. Di Carlo, F. Bouamrane, T. Bouvet, A. J. Durand, M. Kotiranta, V. Krozer, S. Megtert, M. Mineo, and V. Zhurbenko, "Design and Realization Aspects of 1-THz Cascade Backward Wave Amplifier Based on Double Corrugated Waveguide," IEEE Transactions on Electron Devices, vol. 60, no. 3, pp. 1236-1243, Mar. 2013.

[10] C. Paoloni and M. Mineo, "Double Corrugated Waveguide for G-Band Traveling Wave Tubes,", IEEE Trans. on Electron Devices, vol.61, no.12, pp.4259,4263, Dec. 2014.

[11] M. Kotiranta, V. Krozer, and V. Zhurbenko, "Square helix TWT for $\mathrm{THz}$ frequencies," in 35th International Conference on Infrared, Millimetre, and Terahertz Waves, 2010.

[12] C. Paoloni et al. "W-band TWTs for new generation high capacity wireless networks", Proc. IEEE 17th Int. Vac. Electron. Conf. (IVEC), 2016. 